\title{
Mapping the Dynamic Recruitment of Spinal Neurons during Fictive Locomotion
}

\author{
${ }^{\circ}$ Vladimir Rancic, ${ }^{\circledR}$ Klaus Ballanyi, and ${ }^{\circledR}$ Simon Gosgnach \\ Department of Physiology, University of Alberta, Edmonton, Alberta T6G 2E1, Canada
}

The basic rhythmic activity that underlies stepping is generated by a neural network, situated in the spinal cord, known as the locomotor central pattern generator (CPG). While a series of lesion experiments have demonstrated that the mammalian locomotor CPG is distributed throughout the ventral portion of the caudal spinal cord, the specific transverse distribution of this neural network is unclear. Here we evoke fictive locomotor activity of various frequencies in upright spinal cords prepared from male and female neonatal mice. This preparation enables us to use an imaging approach to identify locomotorrelated cells across the transverse plane of the spinal cord. Results indicate that there is a clear shift in the recruitment of cells toward the ventromedial, and away from the ventrolateral, spinal cord as the frequency of fictive locomotion increases. Surprisingly, the analysis of multiple frequencies of fictive locomotion in the same spinal cord indicates that few neurons are involved in locomotor outputs across multiple speeds. Collectively, these experiments allow us to map the transverse distribution of the locomotor CPG and highlight the pattern of dynamic recruitment that occurs within this neural circuit as the frequency is altered. Our findings are consistent with data indicating that there is a speed-dependent recruitment of interneuronal populations during locomotion and suggest that the locomotor CPG is not a static network, but rather the specific cells recruited vary extensively based on demand.

Key words: CPG; interneuron; locomotion

Significance Statement

In this article, we use an imaging approach to identify all those cells that are rhythmically active at the same frequency as fictive locomotion recorded from the ventral roots of the isolated spinal cord. These experiments allow us to map the distribution of locomotor-related cells across the transverse plane of the spinal cord and identify the recruitment pattern of these cells as the frequency of locomotor outputs is altered. Our results indicate that there are drastic changes in the specific neurons activated at different frequencies and provide support for the concept that the locomotor central pattern generator is a modular network with speed-dependent recruitment of interneuronal components.

\section{Introduction}

The locomotor central pattern generator (CPG) is a neural network situated in the spinal cord of mammals that is capable of producing the intrinsic rhythmic alternation required for stepping, independent of sensory or descending input (for review, see Kiehn, 2016). Since its discovery more than a century ago (Brown, 1911), efforts have been made to identify and characterize the neuronal components of this circuit, with the hopes that

\footnotetext{
Received July 13, 2020; revised 0ct. 19, 2020; accepted Nov. 2, 2020.

Author contributions: S.G. designed research; V.R. performed research; V.R. and S.G. analyzed data; K.B. contributed unpublished reagents/analytic tools; S.G. wrote the paper.

The authors declare no competing financial interests.

This research was supported by the Canadian Institutes for Health Research (Grant MOP 86470; S.G.), the Canadian Foundation for Innovation-Alberta Advanced Technology and Education (S.G., K.B.), and the Edmonton University Hospital Foundation (K.B.). We thank Dr. Ying Zhang (Dalhousie University, Halifax, Nova Scotia, (anada) for constructive criticism on the manuscript.

Correspondence should be addressed to Simon Gosgnach at gosgnach@ualberta.ca.

https://doi.org/10.1523/JNEUROSCI.1885-20.2020

Copyright $\odot 2020$ the authors
}

this will lead to a better understanding of the neuronal basis of stepping, and may result in the identification of potential therapeutic targets following damage to the spinal cord (Barbeau et al., 1999). While a series of lesion experiments have been effective in mapping the rostrocaudal arrangement of the locomotor CPG (Kjaerulff and Kiehn, 1996; Cowley and Schmidt, 1997), and a number of genetically defined neuronal populations that participate in locomotion have been identified (for review, see Goulding, 2009), little is known regarding the specific distribution of this circuit in the transverse plane. Whole-cell recordings of interneuronal activity during fictive locomotion have provided some information on the location of interneuronal components of the locomotor CPG; however, comprehensive attempts to map the distribution of this neural network in the transverse plane have largely relied on antibody stains for activity-dependent markers following a bout of fictive locomotor activity (Kjaerulff et al., 1994; Kjaerulff and Kiehn, 1996; Cina and Hochman, 2000). The results of these studies are difficult to decipher since activity-dependent labels do not differentiate between 
tonically active and rhythmically active neurons. Furthermore, sulforhodamine, one of the activity-dependent labels used, has since been shown to label astrocytes (Nimmerjahn et al., 2004). In addition, recent work indicates that there is likely to be a speed-dependent recruitment of interneuronal populations during locomotor activity, particularly in the commissural pathways, with V2a (Crone et al., 2009; Zhong et al., 2011) and $\mathrm{V} 0_{\mathrm{V}}$ (Talpalar et al., 2013) interneuronal populations required for appropriate left-right alternation during fast, but not slow, locomotor activity. These findings suggest that the locomotor CPG may be a dynamic, rather than a static, network and that the specific neurons recruited likely vary based on taskspecific requirements.

Recently, we devised a preparation that allows us to visually identify, and analyze the activity of, all those cells in the transverse plane that burst in concert with fictive locomotor outputs (Rancic et al., 2019). In the present study, we use this preparation to observe neuronal activity during a locomotor task in real time. These experiments enable us to describe the principles of neuronal recruitment during fictive locomotion by identifying the specific position of locomotor-related (LR) cells in the transverse plane of the lumbar spinal cord and to observe the extent to which the distribution shifts at different speeds of fictive locomotion. Imaging data indicate that at slower speeds locomotorrelated neurons are distributed throughout the ventral spinal cord and that there is a clear medial shift as locomotor speed increases. This pattern of recruitment is seen when all locomotor-related neurons are analyzed as well as when we focus solely on those that are glutamatergic. Finally, analysis of multiple frequencies of fictive locomotion in the same preparation indicates that remarkably few neurons are locomotor related at multiple speeds. Collectively, these experiments allow us to define the transverse distribution of the locomotor CPG and highlight the pattern of dynamic recruitment that occurs within this neural circuit as the frequency is altered.

\section{Materials and Methods}

\section{Animals}

All procedures were in accordance with the Canadian Council on Animal Care and approved by the Animal Welfare Committee at the University of Alberta. Experiments were performed on spinal cords isolated from 63 postnatal day 0 (P0) to $\mathrm{P} 4$ mice of either sex. In addition to wild-type mice, VGlut2 ${ }^{\text {Cre }}$ (catalog \#028863, The Jackson Laboratory; RRID:IMSR_JAX:028863) and ROSA26 ${ }^{\text {tdTomato }}$ (\#007909, The Jackson Laboratory; RRID:IMSR_JAX:007909) strains were crossed, and VGlut $2{ }^{\text {Cre }}$ ROSA2 $6{ }^{\text {tdTomato }}$ offspring were used in some experiments to identify glutamatergic neurons.

After anesthesia via inhalation of isoflurane (delivered with $95 \% \mathrm{O}_{2} /$ $5 \% \mathrm{CO}_{2}$ ), animals were quickly decapitated and eviscerated with sharp scissors, and spinal cords (rostral cervical to caudal sacral segments) were dissected out in a bath containing dissecting artificial CSF (ACSF) composed of the following (in $\mathrm{mM}$ ): $120 \mathrm{NaCl}, 2 \mathrm{KCl}, 11$ glucose, 26 $\mathrm{NaHCO}_{3}, 1.25 \mathrm{NaH}_{2} \mathrm{PO}_{4}, 6 \mathrm{MgSO}_{4}, 0.5 \mathrm{CaCl}_{2}, 3 \mathrm{C}_{3} \mathrm{H}_{3} \mathrm{NaO}_{3}$ (Na-pyruvate), and $5 \mathrm{~N}$-acetyl-L-cysteine, $\mathrm{pH}$ 7.4. Following removal, upright spinal cords were prepared (Rancic et al., 2019).

\section{Electrophysiological recording}

Upright spinal cords were transferred to a recording chamber placed under the objective lens of an upright FV1000 MPE Multiphoton Laser Scanning Microscope (Olympus) and perfused with room temperature oxygenated recording aCSF (rACSF) composed of the following (in mm): $120 \mathrm{NaCl}, 3 \mathrm{KCl}, 11$ glucose, $26 \mathrm{NaHCO}_{3}, 1.25 \mathrm{NaH}_{2} \mathrm{PO}_{4}, 1.25$ $\mathrm{MgSO}_{4}, 2.5 \mathrm{CaCl}_{2}, 3 \mathrm{C}_{3} \mathrm{H}_{3} \mathrm{NaO}_{3}$ (Na-pyruvate), and $5 \mathrm{~N}$-acetyl-L-cysteine, $\mathrm{pH}$ 7.4. Fictive locomotor activity was evoked by the addition of NMDA and 5-hydroxytryptamine (5-HT) creatine sulfate complex to the bath and recorded via extracellular suction electrodes attached to the primarily flexor (L1, L2) lumbar ventral roots (VRs). Electroneurogram (ENG) signals from the VRs were acquired at $10,000 \mathrm{~Hz}$, amplified $(10,000 \times)$, bandpass filtered using a differential amplifier (model 1700, A-M Systems), and integrated (time constant, $50 \mathrm{~ms}$ ) using an MA-821/ RSP module (CWE).

\section{$\mathrm{Ca}^{2+}$ imaging}

Membrane-permeant Cal-520 AM (AAT Bioquest) was pressure injected into the ventral aspect of the sectioned surface of the spinal cord using a patch pipette (tip $\varnothing, 20 \mu \mathrm{m})$ containing $5 \mathrm{~mm} \mathrm{Ca}^{2+}$ dye in $20 \%$ DMSO + pluronic acid, further diluted to $0.5 \mathrm{~mm}$ in rACSF containing no $\mathrm{Ca}^{2+}, \mathrm{Mg}^{2+}$, or glucose. A MaiThai-BB Ti:sapphire femtosecond pulsed laser set to excite at $810 \mathrm{~nm}$ was used for fluorescence excitation, and the free cytosolic neuronal $\mathrm{Ca}^{2+}$ concentration was imaged with an XLUMPlan Olympus $20 \times$ immersion objective (1.0 numerical aperture). Loaded cells within a radius of $\sim 400 \mu \mathrm{m}$ and to a depth of $100 \mu \mathrm{m}$ below the sectioned surface of the spinal cord were imaged at a scan rate of $\sim 3 \mathrm{~Hz}$. To correlate cytosolic $\mathrm{Ca}^{2+}$ oscillations with electrophysiological signals, the two systems were synchronized [with a TTL (transistor-transistor logic) pulse] and aligned before analysis.

\section{Experimental design and statistical analysis}

All mean values are reported \pm SD. Student's $t$ tests or one-way ANOVAs (with post hoc Tukey's tests) with a significance level of 0.05 were used to determine means, which differed significantly. As described previously (Haque et al., 2018), circular statistics (Zar, 1974) were used to generate polar plots and to determine the coupling strength between ventral root pairs.

In all cases, the analysis of $\mathrm{Ca}^{2+}$ traces was performed during a 30$60 \mathrm{~s}$ bout of stable fictive locomotor activity. Since the frequency of image acquisition remained constant across experiments, the "cleanliness" of the traces of $\mathrm{Ca}^{2+}$ activity varied such that slower frequencies of fictive locomotion had much smoother traces than those collected during faster frequencies. This was simply because of the number of data points collected during each oscillation. To assess the oscillation frequency and identify LR cells, all optical signals were expressed as relative fluorescence changes with respect to baseline fluorescence $(\Delta F / F)$, and an algorithm developed in Python software was created and applied. The algorithm was designed to detect fluctuations of pixel intensity across the field of view by applying the power spectrum function. This enabled the primary frequency component of each pixel to be determined since the maximum value of the power spectrum peaks for each pixel corresponded to its primary oscillation frequency. All pixels oscillating at the specific locomotor frequency had identical values generated with the power spectrum function, and their peak values were saved in a $2 \mathrm{D}$ matrix, which was converted into an image with false colors. This image enabled us to visually identify regions that exhibited the same frequency of oscillations as the ventral roots. By overlaying a photomicrograph taken during recording, we would confirm that this region corresponded to a cell in the spinal cord; only those cells in which the primary (i.e., largest) peak in the power spectrum was within $0.05 \mathrm{~Hz}$ of the primary peak in the power spectrum of the ventral roots. In none of the LR cells was there an additional peak in the power spectrum that reached $50 \%$ of the height of the primary peak. We accepted cells in which the primary peak varied by $0.05 \mathrm{~Hz}$ since the sampling frequency for $\mathrm{Ca}^{2+}$ imaging $(2-3 \mathrm{~Hz})$ was slower than that for ventral root recordings $(10 \mathrm{kHz})$. The photomicrographs shown in the figures differ from the series of images recorded and analyzed. In the figures shown, we have adjusted the fluorescence levels so that the cells of interest can be easily identified. For recording, the fluorescence intensity was adjusted to visualize as many cells as possible while making sure not to saturate the signal. For crosscorrelation analysis, the fluorescence intensity values of LR cells were exported, and the data points were compared with ventral root ENG activity (which had been downsampled so that its sampling rate matched that of the $\mathrm{Ca}^{2+}$ imaging data). Python software was then used to assemble cross-correlation matrices in which the color map indicates the strength of correlation between each LR cell and the L2 VRs, as well as 
the strength of correlation between the ipsilateral and contralateral L2 VRs.

To display the neuronal density across the transverse plane, all images were acquired using identical parameters (objective lens, zoom factor), and the midpoint of the central canal was used for alignment to normalize cell position across all spinal cords. Contour plots (or heat maps) were then generated in MATLAB.

\section{Results}

The primary goal of these experiments was to map the location of LR cells across the transverse plane of the spinal cord and to investigate how the recruitment pattern changes as the frequency of fictive locomotion is altered. To this end, we used previously developed criteria (Talpalar et al., 2013) to define slow $(<0.2 \mathrm{~Hz})$, medium $(0.2-0.4 \mathrm{~Hz})$, and fast $(>0.4 \mathrm{~Hz})$ frequencies of fictive locomotion. We classified all rhythmically active cells that burst at the same frequency as the lumbar ventral roots as LR but did not include large, laterally situated cells in our analysis since these were presumed to be motoneurons.

In all experiments, 2-15 $\mu \mathrm{M}$ NMDA and 5-30 $\mu \mathrm{M}$ 5-HT were used to evoke fictive locomotion; however, the specific pharmacological cocktail required to generate the three speeds of fictive locomotor activity varied between preparations. Representative traces and group polar plots constructed from spinal cords exhibiting slow $(n=14$; Fig. $1 A)$, medium $(n=37$; Fig. $1 B)$, and fast $(n=23$; Fig. $1 C)$ locomotor activity illustrate that, in all preparations, left-right alternation could be seen between flexorrelated (i.e., L2) ventral roots. Given the nature of the upright preparation, we did not have access to the extensor-related (i.e., L5) ventral roots.

Once a minimum of $3 \mathrm{~min}$ of fictive locomotion of a consistent frequency was established, the next step was to image the cut surface of the spinal cord to identify all those cells that were rhythmically active at the same frequency as the L1/L2 ventral roots during fictive locomotor activity. Based on the location of our Cal-520 AM injection, we were typically able to image $\mathrm{Ca}^{2+}$ oscillations in the ventral two-thirds of the spinal cord from the lateral extent to the midline.

At all locomotor speeds, LR cells were found across the field of view (Fig. 2A-F); however, with increasing locomotor frequency there was a gradual shift such that a greater number of cells close to the central canal were recruited, while the number located more laterally decreased (Fig. $2 A, F$ ). To quantitate this shift, the spinal cord was divided up into three sectors (lateral, central, and medial; Fig. $2 F$ ), and the total number of LR cells in each was tabulated. At slow fictive locomotor speeds, a mean of $5.0 \pm 2.0 \mathrm{LR}$ cells was located in the lateral sector of the spinal cord, $6.0 \pm 3.8$ in the central third, and $5.1 \pm 2.6$ medially. At medium fictive locomotor speeds, $3.0 \pm 2.6$ were located laterally, $6.3 \pm 3.8$ in the central sector, and $11.0 \pm 5.7$ in the most medial sector, while at fast fictive locomotor frequencies the numbers were $2.2 \pm 2.0,5.4 \pm 2.6$, and $16.3 \pm 5.7$, respectively (Fig. 2G). A one-way ANOVA and post hoc Tukey's test comparing the number of locomotor-related neurons in each sector at each frequency indicated that there were significantly more LR cells in the lateral sector at slow fictive locomotor frequencies (one-way ANOVA: $\mathrm{df}=2, F=6.59, p<0.003$; Tukey's HSD: slow vs medium, $p<0.05$; slow vs fast, $p<0.01$ ), while there was no significant difference in the number of LR cells found in this sector at medium and fast fictive locomotor speeds. In the central sector, there was no significant difference in the number of LR cells at any speed (ANOVA: $\mathrm{df}=2, F=0.34, p=0.71$ ), while there were significantly more LR cells found in the medial sector at the
A

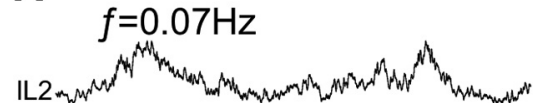

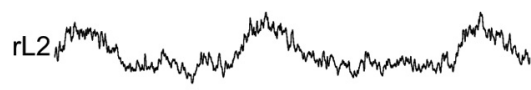

B $f=0.29 \mathrm{~Hz}$
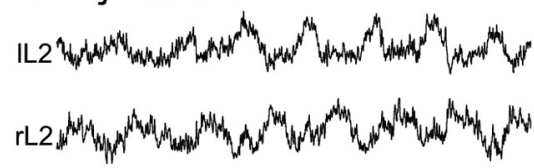

C $f=0.54 \mathrm{~Hz}$
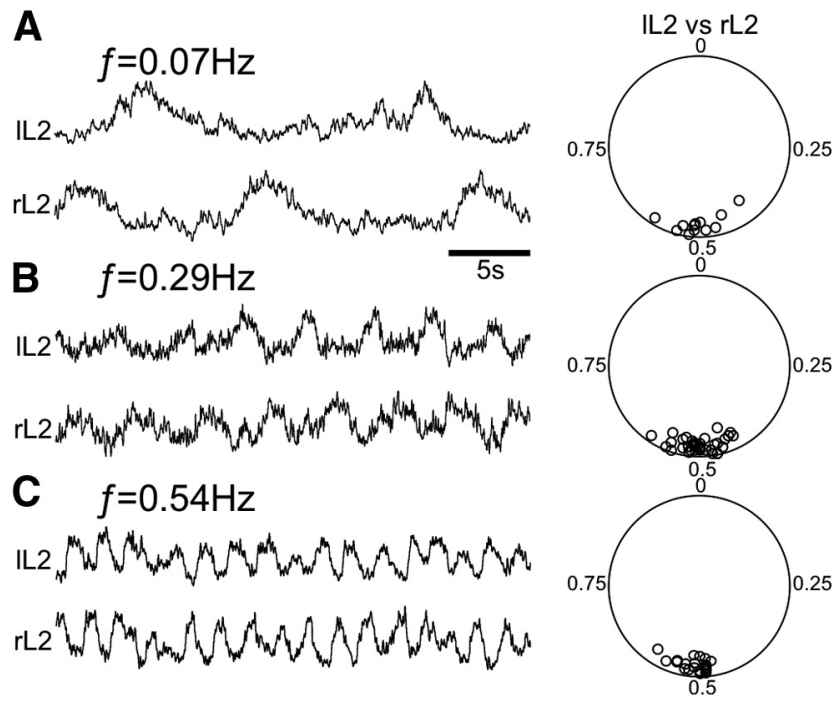

Figure 1. $\boldsymbol{A}-\boldsymbol{C}$, Different frequencies of fictive locomotion evoked with NMDA/5-HT. Slow ( $\boldsymbol{A}$; evoked with $3.9 \mu \mathrm{m}$ NMDA, $10 \mu \mathrm{m}$ 5-HT), medium ( $\boldsymbol{B}$; evoked with $5 \mu \mathrm{m} \mathrm{NMDA}, 10 \mu \mathrm{m}$ 5-HT), and fast (C; evoked with $10 \mu \mathrm{M}$ NMDA, $10 \mu \mathrm{M}$ 5-HT) bouts of fictive locomotion are marked by alternation of left and right flexor-related (L2) ventral root activity. This can be seen in the ENG recordings (left) and group polar plots (right), in which each point represents the vector from an individual experiment.

fastest frequency of fictive locomotion (one-way ANOVA: $\mathrm{df}=2$, $F=23.48, p<0.0001$; Tukey's HSD: slow vs medium, $p<0.01$; slow vs fast, $p<0.001$; medium vs fast, $p<0.01$ ). In addition to this medial shift, there was a significant increase in the total number of LR cells recruited at medium $(23.4 \pm 9.2)$ and fast $(24.9 \pm 7.9)$ frequencies of fictive locomotion when compared with the number recruited during slow $(16.1 \pm 4.1)$ rhythmic activity (one-way ANOVA: $\mathrm{df}=2, F=10.04, p=0.0002$; Tukey's HSD: slow vs medium, $p<0.05$; slow vs fast, $p<0.01$; medium vs fast, nonsignificant; Fig. $2 G$ ). Collectively, these data suggest that the neuronal recruitment patterns between slow fictive locomotor activity vary greatly from those recruited during faster speeds and provide evidence that the locomotor CPG is a dynamic network with distinct groups of neurons involved as the frequency of fictive locomotion changes.

To further investigate the dynamic recruitment pattern of the locomotor CPG, we performed a series of experiments in which we evoked multiple frequencies of fictive locomotion in a single spinal cord. This was achieved by altering the concentrations of 5-HT and NMDA applied to the preparation once a stable bout of fictive locomotion had been established. These experiments enabled us to monitor the change in recruitment away from the lateral (and toward the medial) spinal cord in real time, as the frequency of locomotor activity increased. Furthermore, these experiments allowed us to identify neurons that are locomotor related across multiple frequencies. In eight preparations, we were able to evoke both slow and medium speed fictive locomotion (Fig. 3A). Consistent with our data collected in spinal cords in which fictive locomotor activity was recruited at a single speed, in the slow/medium spinal cords there was a shift in the location of the locomotor-related neurons away from the lateral and toward the medial spinal cord as the frequency of fictive locomotor activity increased (Fig. 3B). The medial shift could also be seen when all locomotor-related neurons from the eight spinal cords at each locomotor frequency were plotted (Fig. 3C, $D)$. To quantitate the shift, we used a paired $t$ test to compare the percentage of locomotor-related neurons that were situated in 
A

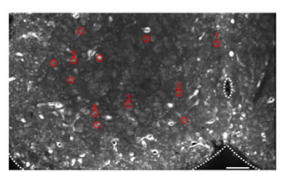

B
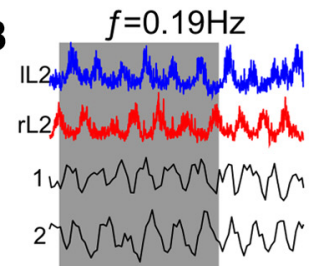

sun Manmorim

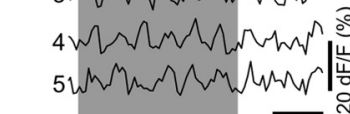

C
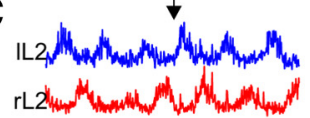

1mingrar MMnMN 3 Mrnm 4 นnn 5 MMnumar

D
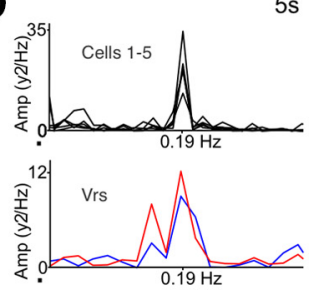

$\mathrm{E}$

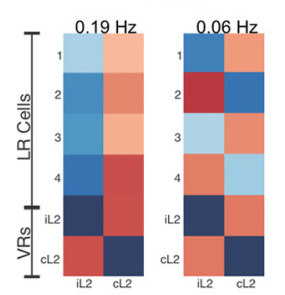

$\mathbf{F}$

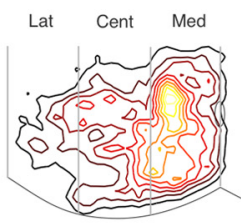

G

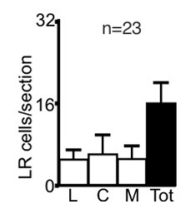

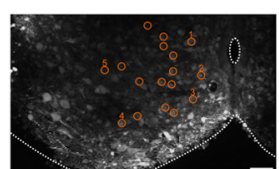

$f=0.31 \mathrm{~Hz}$
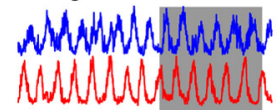

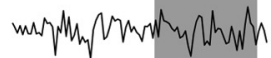

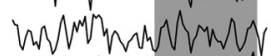

WWMAMTLMN
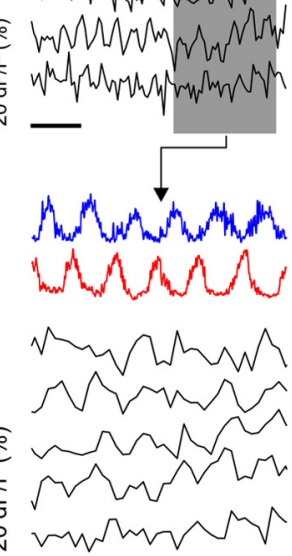

.
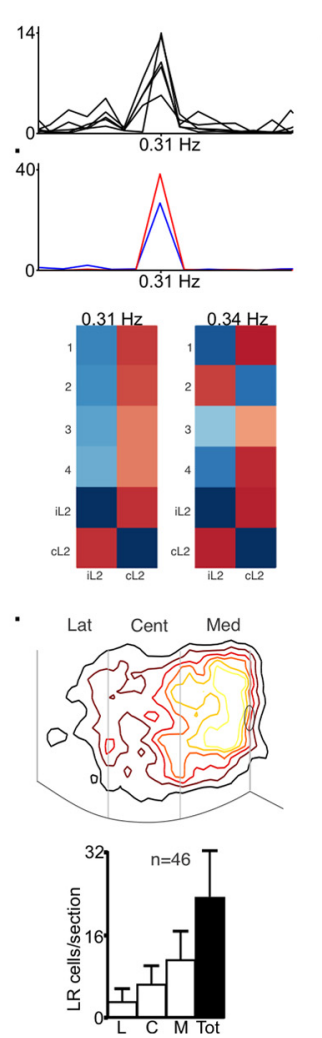

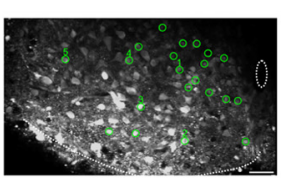

$f=0.43 \mathrm{~Hz}$

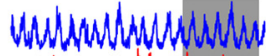

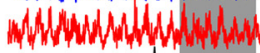

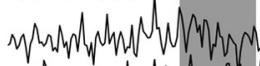

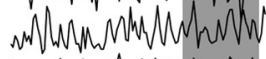
MANMOMOMWOMN

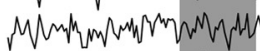

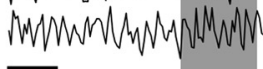
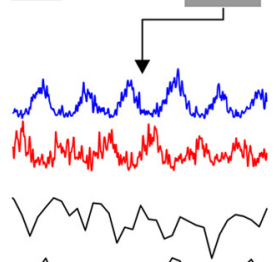
NNm MNMr mins MMNM
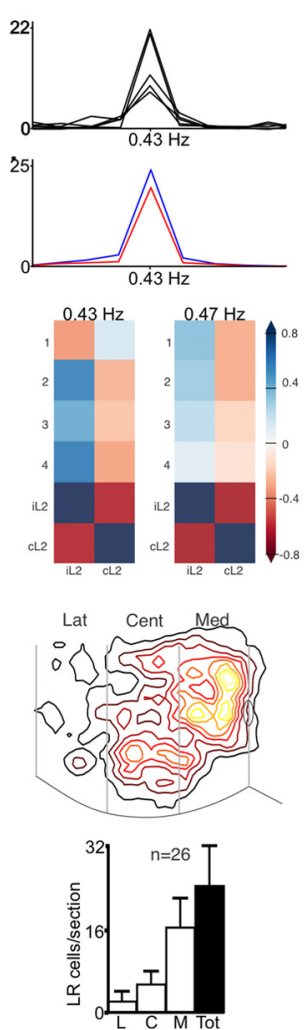

Figure 2. Distribution of locomotor-related cells at different frequencies of fictive locomotion. $\boldsymbol{A}$, Ventral hemi-cords after injection of $\mathrm{Ca}^{2+}$ indicator in which locomotor-related neurons are identified during a bout of slow (red circles), medium (orange circles), and fast (green circles) fictive locomotion. Scale bar, $50 \mu \mathrm{m} . \boldsymbol{B}$, Alternating ENG activity in the left and right $\mathrm{L} 2$ ventral roots, and $\mathrm{Ca}^{2+}$ oscillations (represented as $\triangle F / F$ ) in five locomotor-related cells (specific cells are identified in $\boldsymbol{A}$ ) can be seen during slow (left, evoked with $3 \mu \mathrm{m}$ NMDA, $30 \mu \mathrm{m} 5-\mathrm{HT}$ ), medium (middle, evoked with $2 \mu \mathrm{m} \mathrm{NMDA}, 10 \mu \mathrm{m}$ 5-HT), and fast (right, evoked with $10 \mu \mathrm{m} \mathrm{NMDA}, 7 \mu \mathrm{m}$ 5-HT) bouts of fictive locomotion. C, Shaded region for each set of traces is expanded.

the medial sector during the two speeds of fictive locomotor activity and found that significantly more were situated in this sector as frequency increased (fast, $59.0 \pm 12 \%$; medium, $38.3 \pm 9 \%$; paired $t$ test: $\mathrm{df}=7, t=5.42, p=0.0005, n=8$ spinal cords; Fig. $3 B-$ $D)$. As seen in our previous comparison between medium and slow frequencies of locomotor activity in unique spinal cords (Fig. 2C), the number of locomotor-related neurons recruited during medium speed was significantly greater than the number recruited during slow fictive locomotor activity in the spinal cord in which both speeds were evoked (slow, $13.7 \pm 3.6$ LR cells; medium, $19.7 \pm 5.8$ LR cells; paired $t$ test, $\mathrm{df}=7, t=-2.1, p<0.037$; Fig. 3B,F). A surprisingly small proportion of cells was found to be locomotor related at both speeds ( $2.6 \pm 1.5$ per preparation; Fig. $3 C, E, F$, black dots/bars), and the vast majority of these (17 of 18 in eight spinal cords) were located in the medial sector, close to the central canal, with the remaining cell situated in the central sector (Fig. 3E).

Although it was more subtle, a shift toward the recruitment of cells in the medial spinal cord was also seen in the 10 cords in which we were able to evoke fictive locomotor activity of both medium and fast frequencies (Fig. 4A). In these preparations, we did not observe a significant change in the number of locomotor-related cells between the two frequencies (medium, $24.0 \pm 6.1 \mathrm{LR}$ cells; fast, $22.4 \pm 7.4 \mathrm{LR}$ cells; paired $t$ test, $\mathrm{df}=9, t=-0.69, p=0.25)$; however, the proportion of LR cells situated in the medial sector was greater during faster speeds of fictive locomotion ( $n=10$ spinal cords; fast, $70.6 \pm 10 \%$; medium, $55.0 \pm 8 \%$; paired $t$ test: $\mathrm{df}=9, t=5.72, p=0.00,014$; Fig. $4 B-D$ ). As was seen in the slow/medium spinal cords relatively few cells could be identified as locomotor related at both medium and fast frequencies (4.1 \pm 1.8 ; Fig. $4 C, E, F$, black dots/bars), and those that could were clustered heavily in the most medial sector of the spinal cord (35 of 41 cells in 10 spinal cords) with the remaining 5 in the central sector. These findings support our previous observation of a medial shift as the frequency of locomotor activity increases, and they also indicate that few cells are involved in generating locomotor outputs at multiple speeds.

Several studies have demonstrated that the recruitment of specific genetically defined interneuronal

\footnotetext{
Calibration: $\boldsymbol{B}, 10$ s; $\boldsymbol{C}, 5$ s. D, Spectral analysis indicates that the primary frequency of oscillation for the five oscillatory cells (top plots) is the same as that for the ventral roots (bottom plots) for slow, medium, and fast fictive locomotion. $E$, Cross-correlation matrices in which the activity of four LR cells is related to ipsilateral (iL2) and contralateral (cL2) ventral root activity for two spinal cords during slow, medium, and fast fictive locomotion. Scale to the right applies to all plots, with values of 1.0 indicating completely in phase and -1.0 indicating completely out of phase. $\boldsymbol{F}$, Contour plots indicating the distribution of all locomotor-related cells in multiple spinal cords at slow $(n=23)$, medium $(n=46)$, and fast $(n=26)$ frequency of fictive locomotion. Vertical gray lines divide the hemi-cord into lateral, central, and medial sectors. $\mathbf{G}$, Mean number of LR cells ( \pm SD) per preparation in the lateral $(\mathrm{L})$, central $(\mathrm{C})$, and medial $(\mathrm{M})$ sectors as well as the total number of $L R$ cells (Tot) at each frequency.
} 
A
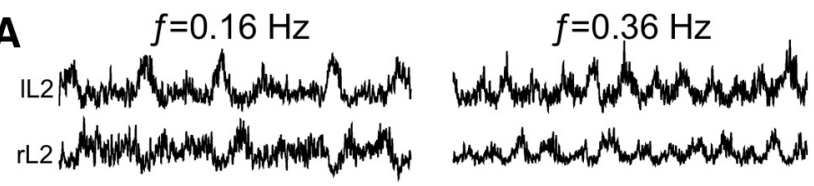

1. M M M M

MNWMMNMN

$2 M \sqrt{n} M M$

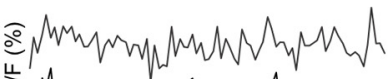

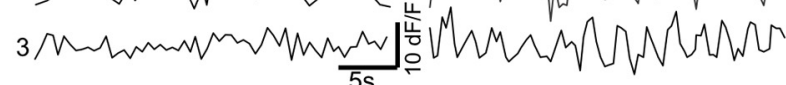

B

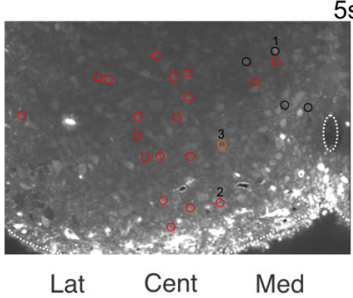

C

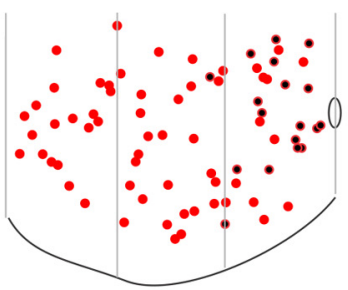

D

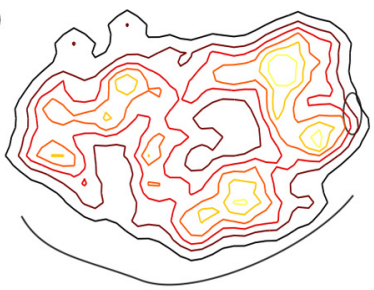

$\mathbf{E}$

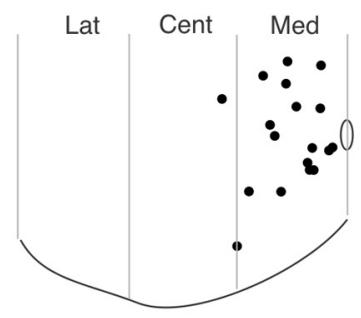

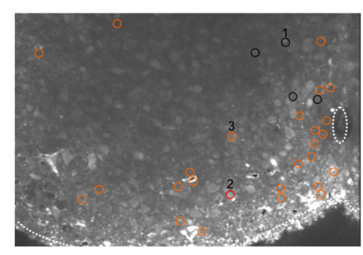

Lat Cent Med $\therefore \therefore \therefore \div$ ‥ $\because \because \because$ $\therefore \quad \cdots$

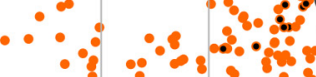

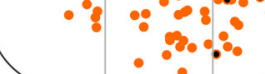

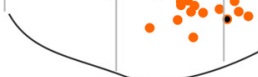

A $f=0.34 \mathrm{~Hz}$ $f=0.55 \mathrm{~Hz}$

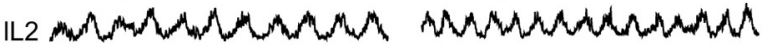

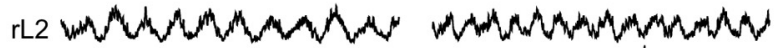

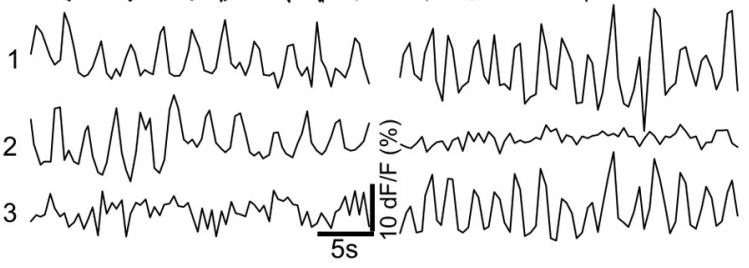

B
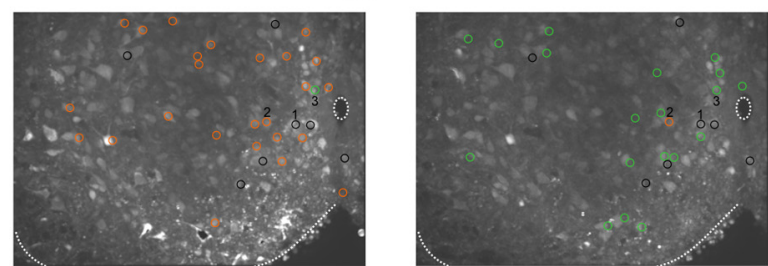

C
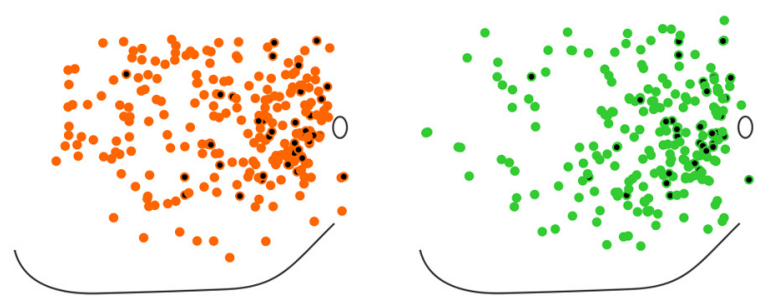

D
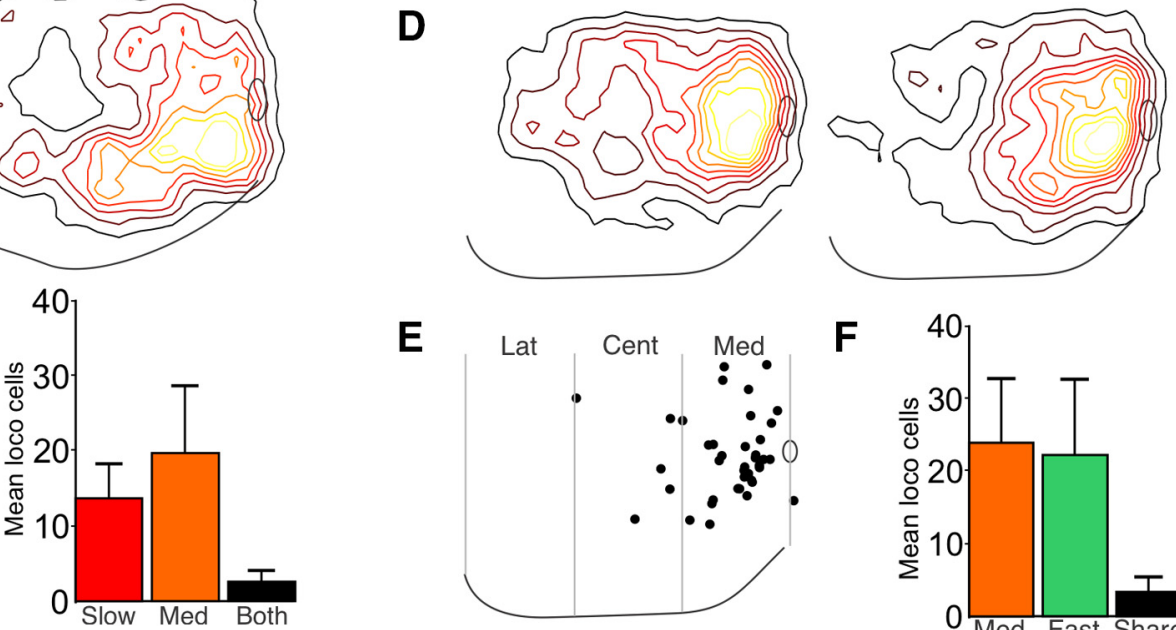

Figure 3. Dynamic changes in recruitment pattern between slow and medium frequencies of fictive locomotion. $A$, Slow (left) and medium (right) speeds of fictive locomotion evoked in the same spinal cord via bath application of 5-HT and NMDA. Immediately below the ventral root recordings from the left (IL2) and right (rL2) ventral roots, $\mathrm{Ca}^{2+}$ activity (represented as $\Delta F / F$ ) in three cells in the ventromedial spinal cord is illustrated. Cell 1 is locomotor related (in phase with rL2) during both slow and medium frequencies of fictive locomotion. Cell 2 is locomotor related (in phase with rL2) during the slow, but not the medium, frequency. (ell 3 is locomotor related (in phase with rL2) during the medium, but not the slow, frequency of fictive locomotion. $\boldsymbol{B}$, Ventral hemi-cords after the injection of $\mathrm{Ca}^{2+}$ indicator in which LR cells are identified during a bout of slow (red circles) and medium (orange circles) fictive locomotion. Those cells that are LR during bouts of both slow and medium frequencies of fictive locomotion are surrounded by a black circle. Scale bar, $50 \mu \mathrm{m}$. Cells 1,2 , and 3 from $\boldsymbol{A}$ are identified in both images. $\boldsymbol{C}, \boldsymbol{D}$, Dot $(\boldsymbol{C}$ ) and contour $(\boldsymbol{D})$ plots indicating the location and distribution of all LR cells at each speed of fictive locomotion in eight spinal cords. Dots with black centers indicate those cells that are LR at both speeds. $\boldsymbol{E}$, Dot plot indicating that all cells that were $L R$ at both slow and medium frequencies of fictive locomotion in the eight spinal cords investigated. Vertical gray lines divide the hemi-cord into lateral, central, and medial sectors. $\boldsymbol{F}$, Mean number of LR cells ( \pm SD) per preparation in spinal cords locomoting at slow (red) and medium (orange) frequencies as well as the mean number that are LR at both speeds (black).
$\mathbf{E}$
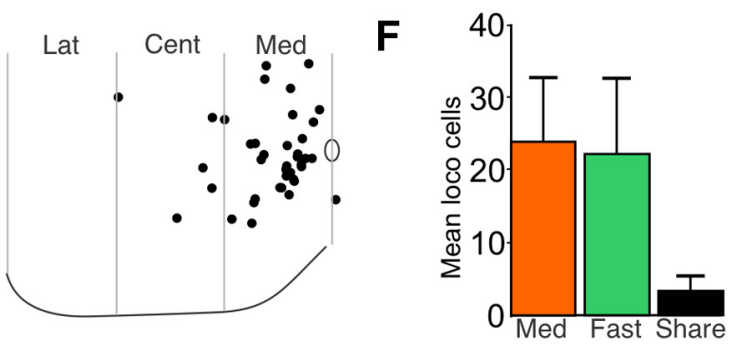

Figure 4. Dynamic changes in recruitment pattern between medium and fast frequencies of fictive locomotion. $A$, Medium (left) and fast (right) speeds of fictive locomotion evoked in the same spinal cord via bath application of 5-HT and NMDA. Immediately below the ventral root recordings from the left (IL2) and right (rL2) ventral roots, $\mathrm{Ca}^{2+}$ activity (represented as $\Delta F / F$ ) in three LR cells in the ventromedial spinal cord is illustrated. Cell 1 bursts in phase with IL2 during both medium and fast frequencies of fictive locomotion. Cell 2 bursts in phase with rL2 during the medium but not the fast frequency. Cell 3 bursts in phase with IL2 during the fast, but not the medium, frequency of fictive locomotion. $\boldsymbol{B}$, Ventral hemi-cords after the injection of $\mathrm{Ca}^{2+}$ indicator. LR cells are indicated by orange (left-medium frequency) or green (right-fast frequency) circles with cells that are $L R$ at both frequencies surrounded by a black circle. Scale bar, $50 \mu \mathrm{m}$. Cells 1,2 , and 3 from $A$ are identified in both images. $\boldsymbol{C}, \boldsymbol{D}, \operatorname{Dot}(\boldsymbol{C})$ and contour $(\boldsymbol{D})$ plots indicating the location and distribution of all $L R$ cells at either speed of fictive locomotion. Dots with black centers indicate those cells that are LR at both speeds. $\boldsymbol{E}$, Dot plot indicating that all cells that were $L R$ at both medium and fast frequencies of fictive locomotion ( $n=10$ spinal cords). $\boldsymbol{F}$, Mean number of LR cells $( \pm S D)$ per preparation in spinal cords locomoting at medium (orange) and fast (green) frequencies as well as the mean number that are $L R$ at both speeds (black). 


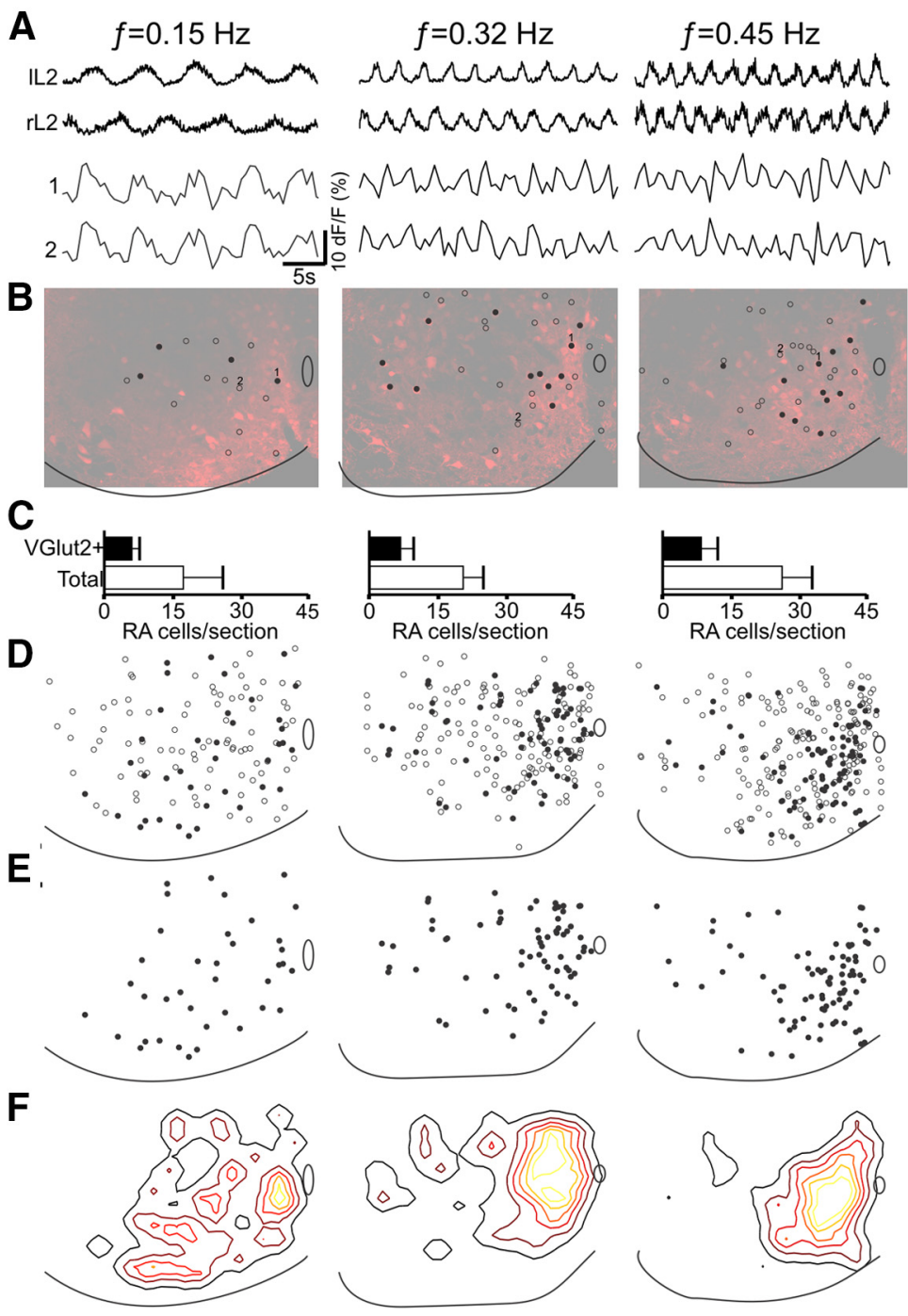

Figure 5. Recruitment pattern of glutamatergic neurons is altered as locomotor speed increases. $\boldsymbol{A}$, Slow (left), medium (middle), and fast (right) fictive locomotion (evoked via bath application of 5-HT and NMDA) in spinal cords from VGlut2 ${ }^{\text {Cre }}:$ ROSA26 ${ }^{\text {tdtomato }}$ mice. Below the ENG recordings from left (IL2) and right (rL2) $\mathrm{Ca}^{2+}$ activity (represented as $\triangle F / F$ ) from two LR cells is illustrated. For each frequency of fictive locomotion cell 1 is VGlut2 ${ }^{+}$and cell 2 is VGlut2 ${ }^{-}$. B , Ventral hemi-cords of VGlut2 ${ }^{\text {Cre }}:$ ROSA26 ${ }^{\text {tdtomato }}$ mice in which VGlut2 ${ }^{+}$cells appear red. LR cells are identified during a bout of fictive locomotion, with open circles indicating VGlut2 $2^{-} L R$ cells and filled circles indicated VGlut2 $^{+}$LR cells. Scale bar, $50 \mu \mathrm{m}$. Cells 1 and 2 from $\boldsymbol{A}$ are indicated for each frequency of fictive locomotion. $\boldsymbol{C}$, Bar graphs indicating the mean number of $L R$ cells as well as VGlut2 ${ }^{+} L R$ cells at each locomotor frequency $(n=10$ for each fictive locomotor frequency). $\boldsymbol{D}, \boldsymbol{E}$, Dot plots indicating the distribution of all $L R$ cells at each speed of fictive locomotion $(\boldsymbol{D})$ in which VGlut2 ${ }^{+}$LR cells are indicated by filled circles and VGlut2 ${ }^{-}$by open circles; VGlut2 ${ }^{+}$cells alone are illustrated in $\boldsymbol{E}$ to draw attention to the medial shift in the recruitment of these cells as fictive locomotor speed increases. $\boldsymbol{F}$, Contour plot to accompany $\boldsymbol{E}$, which highlights the distribution of VGlut2 ${ }^{+}$LR cells.

populations that participate in locomotor activity vary based on speed. The inhibitory $\mathrm{V} 0_{\mathrm{D}}$ neurons facilitate left-right alternation at slow locomotor speeds while the excitatory $\mathrm{V}_{\mathrm{V}}$ and $\mathrm{V} 2 \mathrm{a}$ populations become involved as the frequency of locomotion increases (Talpalar et al., 2013). While we did not have access to transgenic mouse lines in which these specific interneuronal populations could be visually identified, we were interested in determining whether there is an overall shift in the recruitment of excitatory neurons as fictive locomotor frequency increases. To accomplish this, we conducted imaging experiments during fictive locomotion in upright spinal cords prepared from VGlut2 ${ }^{\mathrm{Cre}}: \mathrm{R} 26^{\text {tdtomato }}$ mice in which glutamatergic neurons express a reporter protein and can be identified during the recording (Fig. 5A,B). For these experiments, the total number of LR cells were identified, as were the total number of glutamatergic LR cells in VGlut2 ${ }^{\mathrm{Cre}}$ : $\mathrm{R} 26^{\text {tdtomato }}$ spinal cords during fictive locomotion at slow ( $n=10$ spinal cords), medium ( $n=10$ spinal cords), and fast ( $n=10$ spinal cords) frequencies. Consistent with our previous findings, we saw an increased overall number of LR cells at medium and fast frequencies when compared with the number recruited during slow fictive locomotion (slow, 12.4 \pm 6.8 cells; medium, $21.1 \pm 4.5$ cells; fast, 24 . \pm 5.2 cells; one-way ANOVA: $\mathrm{df}=2, F=11.71, p=0.0002$; Tukey's HSD: slow vs medium, $p<0.01$; slow vs fast, $p<0.01$; fast vs medium, nonsignificant; Fig. $5 C$ ) as well as a shift in the recruitment of neurons toward the midline as the frequency of fictive locomotion increased (Fig. 5B,D). The percentage of total LR cells that were VGlut2 ${ }^{+}$remained remarkably consistent across all three frequencies, comprising $34 \pm 6 \%$ of the total population at slow locomotor speeds, $34 \pm 8 \%$ at medium locomotor speeds, and $33 \pm 9 \%$ during fast fictive locomotion (one-way ANOVA: $\mathrm{df}=2, F=0.03$, $p=0.97$; Fig. $5 C, E$ ). Despite the similarity in the percentages of LR cells that were glutamatergic, there was a clear shift toward the recruitment of glutamatergic LR cells in the medial sector of the spinal cord at higher frequencies of fictive locomotion with only $42 \pm 12 \%$ of the LR glutamatergic cells residing in this region at the slowest frequency of fictive locomotion, $67 \pm 7 \%$ at medium speed, and $76 \pm 9 \%$ during high-speed fictive locomotion (one-way ANOVA: $\mathrm{df}=2$, $F=34.18, p<0.0001$; Tukey's HSD: slow vs medium, $p<0.01$; slow vs fast, $p<0.01$; medium vs fast, nonsignificant). Together, these data indicate that the pattern of recruitment of excitatory neurons mirrors the overall recruitment pattern as locomotor speed is modulated, and that the overall proportion of glutamatergic neurons recruited remains remarkably consistent across locomotor frequencies.

\section{Discussion}

This study is the first to glimpse inside the mammalian spinal cord during different speeds of fictive locomotor activity, and to identify the distribution and recruitment patterns of the specific cells that participate. Our results build on the pioneering work of the Kiehn laboratory, which used a series of lesions to the isolated spinal cord during fictive locomotion to demonstrate that the key components of the locomotor CPG are situated in the ventral spinal cord with a rostral to caudal gradient (Kjaerulff and Kiehn, 1996). By using the upright spinal cord preparation and altering the speed of fictive locomotor activity, we were able to (1) provide additional insight into the structure of this neural circuit by mapping the location of the cells that participate in fictive locomotor activity in the transverse plane, (2) demonstrate how the recruitment of cells shifts medially as the speed of fictive locomotor activity increases, and (3) reveal that the locomotor CPG is an extremely diverse network with few neurons involved during multiple fictive locomotor speeds. 
Before this study, work incorporating a whole-cell recording approach has provided some insight into the location of locomotor-related cells; however, these studies typically focused on a specific region of the spinal cord or a specific cell population, and do not provide a comprehensive overview of the distribution in the transverse plane. In addition, a number of groups have used an activity-dependent labeling approach to investigate the transverse distribution of the mammalian locomotor CPG after a bout of either real or fictive locomotion (Jasmin et al., 1994; Kjaerulff et al., 1994). The results of these studies indicate that LR cells are spread across the ventral spinal cord (for review, see Kiehn and Kjaerulff, 1998); however, there are serious flaws with the interpretation of this work since the reliability of many of the markers used has since been questioned. False negatives (Jasmin et al., 1994; Kjaerulff et al., 1994) and false positives (Carr et al., 1995) have been shown to be extremely common when working with activity-dependent labels and, perhaps more importantly, these markers do not have the capability to distinguish between rhythmically active and tonically active neurons. This latter point is a serious concern since, in the present study, we were able to identify a substantial number of cells in each focal plane that were either tonically active or rhythmically active at a frequency either much faster or slower than fictive locomotion.

By using a $\mathrm{Ca}^{2+}$ imaging approach we were able to selectively focus on those cells that burst at the same frequency as the ventral roots during a bout of fictive locomotion. Before this study, several attempts have been made to image rhythmically active LR cells during fictive locomotion in the mammalian (Nakayama et al., 2002; Wilson et al., 2007; Antri et al., 2011; Hinckley and Pfaff, 2013) or avian (O’Donovan et al., 2005) spinal cord. While false positives are highly unlikely with $\mathrm{Ca}^{2+}$ imaging, the underreporting of LR cells because of poor loading with the $\mathrm{Ca}^{2+}$ indicator make false negatives (i.e., "missing" LR cells) a possibility. This raises the prospect that the number of LR cells is greater than that reported here; however, we believe that we have minimized the chances of this occurring by standardizing both the injection site, as well as the quantity of $\mathrm{Ca}^{2+}$ indicator applied. Furthermore, our development of an analysis algorithm in Python, which identifies fluctuations in pixel intensity, standardized the process, enabled the identification of oscillatory cells that could not be discerned by eye, and thus minimized the chances of human error. Despite this, the proportion of cells shown to be locomotor related here is lower than what is seen in whole-cell recording studies (Kiehn et al., 1996; Raastad et al., 1996; Butt and Kiehn, 2003; Quinlan and Kiehn, 2007), indicating that we may not be capturing all LR cells using this imaging approach. It is important to keep in mind, however, that electrophysiological experiments have an inherent sampling bias in that only healthy cells, those cells belonging to a specific population, or those cells within a specific region of the spinal cord are targeted, as opposed to the present study, which did not discriminate.

Another caveat with the present study is that some of the cells deemed to be locomotor related may be glia rather than neurons. Glia cells tend to be small compared with neurons, and, based on the size of the cells seen to exhibit $\mathrm{Ca}^{2+}$ oscillation in this study, together with our previous unpublished observation that cells in the spinal cord with high membrane resistance and extremely hyperpolarized values of resting membrane potential (presumably glia) do not oscillate during fictive locomotion, we do not expect glia to comprise a substantial proportion of our dataset. Furthermore, although some glia cells in higher centers have been shown to oscillate (Alvarez-Maubecin et al., 2000; Amzica et al., 2002), the depolarizations were in the range of
0.5-3 $\mathrm{mV}$ and were too small to evoke the large $\mathrm{Ca}^{2+}$ responses observed in this study.

One of the primary findings of this study is the significant shift in the location of all LR cells, as well as of those glutamatergic LR cells, toward the medial spinal cord as the frequency of fictive locomotion increases. Although it is possible that the recruitment pattern seen in this study may have been influenced by the increased concentrations of NMDA typically used to evoke higher frequencies of fictive locomotion, we do not believe that this had a substantial effect since the same recruitment pattern was seen in cases where low doses of NMDA were able to evoke fast locomotor frequencies. Furthermore, if a higher concentration of NMDA were to increase depolarization, we would expect it to affect all cells across the spinal cord, as opposed to those specifically located medially. This medial shift as the frequency of fictive locomotor activity increases thus provides support for the idea that the locomotor CPG is a modular network with speed-dependent recruitment of interneuronal components, as has been previously suggested (Bellardita and Kiehn, 2015). A speed-dependent shift in neuronal recruitment is also seen in the zebrafish, where excitatory interneurons located in the ventralmost regions of the cord are rhythmically active at the slowest swimming speeds, and more dorsally located neurons become recruited as frequency increases (McLean et al., 2007, 2008). The same study indicated the opposite recruitment pattern (dorsal to ventral as speed increases) for inhibitory interneurons. While the direction of the shift that we see in the mouse spinal cord is different from that in the zebrafish, our findings raise the possibility that a gradual change in the position of the specific neurons recruited as the frequency of locomotor activity increases may be a mechanism conserved from early spinal cord development.

Over the past 2 decades, a molecular analysis of developing spinal interneurons has resulted in the identification, and functional characterization, of interneuronal components of the locomotor CPG. Accordingly, we now know the general function of the four genetically distinct parent populations of spinal interneurons (V0, V1, V2, V3), which originate in the ventral spinal cord (Goulding, 2009). Recently, each parent population has been further subdivided into multiple subsets, and it has become apparent that some of these are recruited at specific speeds of locomotion (Kiehn, 2016). Perhaps the best example of this involves the circuitry coordinating left-right alternation across the midline of the spinal cord. As the frequency of locomotor activity increases, the excitatory $\mathrm{V} 2 \mathrm{a}$ and $\mathrm{V} 0_{\mathrm{V}}$ interneuronal populations become involved in coordinating left-right alternation, and reliance on the inhibitory $\mathrm{V} 0_{\mathrm{D}}$ cells, which are essential for this function at slower locomotor speeds, is reduced (Crone et al., 2009; Talpalar et al., 2013). In the present study, we used the VGlut $2{ }^{\text {Cre }}$ ROSA2 $66^{\text {tdtomato }}$ mouse to determine whether there was a general trend toward the recruitment of excitatory neurons as the speed of fictive locomotion increased. Surprisingly, the specific proportion of excitatory LR cells remained remarkably consistent even as the recruitment pattern shifted toward the medial sector of the spinal cord with increasing locomotor frequencies. The most straightforward explanation for this is that the increased recruitment of the excitatory $\mathrm{V} 2 \mathrm{a}$ and $\mathrm{V0} \mathrm{V}_{\mathrm{V}}$ neurons coincides with an increase in the recruitment of other, medially situated, inhibitory neurons. This would also account for our finding that an increased number of LR cells can be identified at faster speeds of fictive locomotion. Based on what we know regarding neurotransmitter phenotype of genetically defined interneuronal populations, and their distribution pattern in the medial spinal cord, two excellent candidates for recruitment as 
locomotor frequency increases are the primarily inhibitory dI6 and $\mathrm{V} 2 \mathrm{~b}$ interneuronal populations. In addition, although we cannot quantify the specific number of GABAergic or glycinergic neurons that were locomotor related in this study, our finding that the proportion of LR cells that are glutamatergic remains stable regardless of frequency raises the possibility that the production of locomotor activity may rely on a precise balance in the ratio of excitatory and inhibitory neurons involved [i.e., constant excitation/inhibition (or E/I ratio)], a general principle thought to be essential for neural network function and stability throughout the CNS (Okun and Lampl, 2008; Atallah and Scanziani, 2009; Yizhar et al., 2011; Froemke, 2015; Denève and Machens, 2016; Bhatia et al., 2019).

Another surprising finding from this study was the rarity with which we observed a cell to be locomotor related during multiple frequencies of fictive locomotor activity. Overall, only $10-20 \%$ of LR cells fit this description when multiple frequencies were evoked in the same spinal cord. Although it is possible that slight movements of the preparation that we observed (primarily in the $z$ plane) resulted in cells moving out of the focal plane and thus not being able to be assessed during multiple frequencies, the vast majority of cells could be identified at both speeds of fictive locomotion (Figs. 3B, $4 B$ ), and we think that this did not significantly impact our findings. Instead, we suggest that a built-in redundancy exists such that the specific cells that participate in locomotor activity are not as important as the cell type and connectivity of those recruited. This would result in the specific members of a genetically defined population that participates in locomotor activity to vary from bout to bout, and as long as a certain proportion of a given population is locomotor related, it is sufficient to generate the required rhythmic, alternating outputs. This redundancy would account for studies that have investigated rhythmicity in genetically defined interneuronal populations (via whole-cell recording) and have shown that only one-half to two-thirds of the members of a population are rhythmically active and locomotor related (Zhong et al., 2011; Dougherty et al., 2013).

Over the past few years, it has come to light that the locomotor CPGs in both aquatic (McLean et al., 2007, 2008; Ampatzis et al., 2014) and limbed (Bellardita and Kiehn, 2015) species are likely to have a sequential and modular design such that an increase in locomotor speed is achieved by the activation of distinct microcircuits. While there is a clear disconnect between different frequencies of fictive locomotion and the different gaits that are revealed at specific speeds of overground stepping in the intact animal, the present study sheds light into the specific recruitment pattern of neurons as the frequency of locomotion changes and may provide insight into the manner in which various modules are activated during a locomotor task.

\section{References}

Alvarez-Maubecin V, García-Hernández F, Williams JY, Van B (2000) Functional coupling between neurons and glia. J Neurosci 20:4091-4098.

Ampatzis K, Song J, Ausborn J, El Manira A (2014) Separate microcircuit modules of distinct v2a interneurons and motoneurons control the speed of locomotion. Neuron 83:934-943.

Amzica F, Massimini M, Manfridi A (2002) Spatial buffering during slow and paroxysmal sleep oscillations in cortical networks of glial cells in vivo. J Neurosci 22:1042-1053.

Antri M, Mellen N, Cazalets JR (2011) Functional organization of locomotor interneurons in the ventral lumbar spinal cord of the newborn rat. PLoS One 6:e20529.

Atallah BV, Scanziani M (2009) Instantaneous modulation of gamma oscillation frequency by balancing excitation with inhibition. Neuron 62:566-577.
Barbeau H, McCrea DA, O’Donovan MJ, Rossignol S, Grill WM, Lemay MA (1999) Tapping into spinal circuits to restore motor function. Brain Res Brain Res Rev 30:27-51

Bellardita C, Kiehn O (2015) Phenotypic characterization of speed-associated gait changes in mice reveals modular organization of locomotor networks. Curr Biol 25:1426-1436.

Bhatia A, Moza S, Bhalla US (2019) Precise excitation-inhibition balance controls gain and timing in the hippocampus. Elife 8:e43415.

Brown TG (1911) The intrinsic factors in the act of progression in the mammals. Proc R Soc Lond B 84:308-319.

Butt SJ, Kiehn O (2003) Functional identification of interneurons responsible for left-right coordination of hindlimbs in mammals. Neuron 38:953963.

Carr PA, Huang A, Noga BR, Jordan LM (1995) Cytochemical characteristics of cat spinal neurons activated during fictive locomotion. Brain Res Bull $37: 213-218$

Cina C, Hochman S (2000) Diffuse distribution of sulforhodamine-labeled neurons during serotonin-evoked locomotion in the neonatal rat thoracolumbar spinal cord. J Comp Neurol 423:590-602.

Cowley KC, Schmidt BJ (1997) Regional distribution of the locomotor pattern-generating network in the neonatal rat spinal cord. J Neurophysiol 77:247-259.

Crone SA, Zhong G, Harris-Warrick R, Sharma K (2009) In mice lacking V2a interneurons, gait depends on speed of locomotion. J Neurosci 29:7098-7109.

Denève S, Machens CK (2016) Efficient codes and balanced networks. Nat Neurosci 19:375-382.

Dougherty KJ, Zagoraiou L, Satoh D, Rozani I, Doobar S, Arber S, Jessell TM, Kiehn O (2013) Locomotor rhythm generation linked to the output of spinal shox2 excitatory interneurons. Neuron 80:920-933.

Froemke RC (2015) Plasticity of cortical excitatory-inhibitory balance. Annu Rev Neurosci 38:195-219.

Goulding M (2009) Circuits controlling vertebrate locomotion: moving in a new direction. Nat Rev Neurosci 10:507-518.

Haque F, Rancic V, Zhang W, Clugston R, Ballanyi K, Gosgnach S (2018) WT1-expressing interneurons regulate left-right alternation during mammalian locomotor activity. J Neurosci 38:5666-5676.

Hinckley CA, Pfaff SL (2013) Imaging spinal neuron ensembles active during locomotion with genetically encoded calcium indicators. Ann N Y Acad Sci 1279:71-79.

Jasmin L, Gogas KR, Ahlgren SC, Levine JD, Basbaum AI (1994) Walking evokes a distinctive pattern of Fos-like immunoreactivity in the caudal brainstem and spinal cord of the rat. Neuroscience 58:275-286.

Kiehn O (2016) Decoding the organization of spinal circuits that control locomotion. Nat Rev Neurosci 17:224-238.

Kiehn O, Kjaerulff O (1998) Distribution of central pattern generators for rhythmic motor outputs in the spinal cord of limbed vertebrates. Ann N Y Acad Sci 860:110-129.

Kiehn O, Johnson BR, Raastad M (1996) Plateau properties in mammalian spinal interneurons during transmitter-induced locomotor activity. Neuroscience 75:263-273.

Kjaerulff O, Kiehn O (1996) Distribution of networks generating and coordinating locomotor activity in the neonatal rat spinal cord in vitro: a lesion study. J Neurosci 16:5777-5794.

Kjaerulff O, Barajon I, Kiehn O (1994) Sulphorhodamine-labelled cells in the neonatal rat spinal cord following chemically induced locomotor activity in vitro. J Physiol 478:265-273.

McLean DL, Fan J, Higashijima S, Hale ME, Fetcho JR (2007) A topographic map of recruitment in spinal cord. Nature 446:71-75.

McLean DL, Masino MA, Koh IY, Lindquist WB, Fetcho JR (2008) Continuous shifts in the active set of spinal interneurons during changes in locomotor speed. Nat Neurosci 11:1419-1429.

Nakayama K, Nishimaru H, Kudo N (2002) Basis of changes in left-right coordination of rhythmic motor activity during development in the rat spinal cord. J Neurosci 22:10388-10398.

Nimmerjahn A, Kirchhoff F, Kerr JN, Helmchen F (2004) Sulforhodamine 101 as a specific marker of astroglia in the neocortex in vivo. Nat Methods 1:31-37.

O’Donovan MJ, Bonnot A, Wenner P, Mentis GZ (2005) Calcium imaging of network function in the developing spinal cord. Cell Calcium 37:443450 . 
Okun M, Lampl I (2008) Instantaneous correlation of excitation and inhibition during ongoing and sensory-evoked activities. Nat Neurosci 11:535-537.

Quinlan KA, Kiehn O (2007) Segmental, synaptic actions of commissural interneurons in the mouse spinal cord. J Neurosci 27:6521-6530.

Raastad M, Johnson BR, Kiehn O (1996) The number of postsynaptic currents necessary to produce locomotor-related cyclic information in neurons in the neonatal rat spinal cord. Neuron 17:729-738.

Rancic V, Haque F, Ballanyi K, Gosgnach S (2019) Using an upright preparation to identify and characterize locomotor related neurons across the transverse plane of the neonatal mouse spinal cord. J Neurosci Methods 323:90-97.

Talpalar AE, Bouvier J, Borgius L, Fortin G, Pierani A, Kiehn O (2013) Dualmode operation of neuronal networks involved in left-right alternation. Nature 500:85-88.
Wilson JM, Dombeck DA, Díaz-Ríos M, Harris-Warrick RM, Brownstone RM (2007) Two-photon calcium imaging of network activity in XFPexpressing neurons in the mouse. J Neurophysiol 97:3118-3125.

Yizhar O, Fenno LE, Prigge M, Schneider F, Davidson TJ, O'Shea DJ, Sohal VS, Goshen I, Finkelstein J, Paz JT, Stehfest K, Fudim R, Ramakrishnan C, Huguenard JR, Hegemann P, Deisseroth K (2011) Neocortical excitation/inhibition balance in information processing and social dysfunction. Nature 477:171-178.

Zar JH (1974) Circular distribution. In: Biostatistical analysis, pp 310-327. Englewood Cliffs, NJ: Prentice Hall.

Zhong G, Sharma K, Harris-Warrick RM (2011) Frequency-dependent recruitment of V2a interneurons during fictive locomotion in the mouse spinal cord. Nat Commun 2:274 